\title{
Complete response and long-term survival of leptomeningeal carcinomatosis from breast cancer with maintenance endocrine therapy
}

\author{
Muneera Majed Almajed, ${ }^{1,2}$ Khashayar Esfahani, ${ }^{1}$ Manuela Pelmus, ${ }^{3}$ Lawrence Panasci $^{1}$
}

${ }^{1}$ Jewish General Hospital, McGill University, Montreal, Quebec, Canada

${ }^{2}$ Oncology Department, Prince Sultan Military Medical City,

Riyadh, Saudi Arabia

${ }^{3}$ Pathology Department, Jewish

General Hospital, McGill University, Montreal, Quebec, Canada

\section{Correspondence to} Dr Muneera Majed Almajed, mmalmajedi@gmail.com

Accepted 15 May 2016

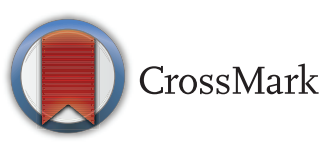

To cite: Almajed MM, Esfahani K, Pelmus M, et al. BMJ Case Rep Published online: [please include Day Month Year] doi:10.1136/bcr2016-215525

\section{SUMMARY}

Leptomeningeal carcinomatosis carries a poor prognosis in breast cancer. Treatment modalities are geared towards tumour molecular characteristics, as well as symptoms and patient performance status. It has previously been postulated that endocrine treatments used for the treatment of metastatic breast cancer do cross the blood-brain barrier and can achieve antineoplastic effects in the central nervous system. We report a case of metastatic breast cancer in a 65-year-old woman who developed leptomeningeal carcinomatosis. She was initially treated with intrathecal methotrexate, which was stopped due to toxicity, followed by maintenance endocrine therapy. She achieved a sustained complete radiological and cerebrospinal fluid cytological response for over 9 years. She eventually passed away of ischaemic bowel unrelated to her cancer.

\section{BACKGROUND}

Occurrence of leptomeningeal carcinomatosis (LC) is a rare manifestation of hormone-positive breast cancer. It is more often associated with human epidermal growth factor receptor 2 (HER2)+, triple negative and the lobular variant of hormonepositive breast cancer. Estimates of the incidence of LC in patients with breast cancer in clinical series range from $1 \%$ to $8 \%$, with autopsy series revealing an incidence as high as $16 \% .^{12}$ The prognosis is usually poor with the median survival in untreated patients with leptomeningeal metastasis ranging from 6 to 8 weeks. For treated patients, the prognosis is widely variable, with prospective studies showing median overall survival times of 9-30 weeks. ${ }^{12}$

Treatment modalities for LC include surgery for obstructive hydrocephalus, radiation therapy, intravenous chemotherapy or intrathecal (IT) chemotherapy. The initial treatment choice depends on the molecular characteristics of the breast cancer, the presence of symptoms, patient performance status, extent of active systemic disease, the availability of systemic treatment options and patient preference.

The role of hormonal therapy for LC in breast cancer is still unclear. Several case reports suggest their efficacy at treating LC as stand-alone drugs, although no prospective trials have been conducted to validate this effect. The results from these case reports have raised the possibility that most antihormonal therapies have the potential to cross the blood-brain barrier, resulting in clinically significant tumour response.
We present a case of successful long-term remission of LC with antihormonal therapy in a patient who could not tolerate IT methotrexate (ITMx). Her 9 years of central nervous system (CNS) diseasefree survival is the longest currently reported in the literature.

\section{CASE PRESENTATION}

A 37-year-old woman had been diagnosed, in 1988, with a right-sided $1.2 \mathrm{~cm}$ low-grade invasive ductal carcinoma. At the time, her tumour was strongly positive for oestrogen and progesterone staining. She underwent segmental mastectomy and sentinel node biopsy, followed by postoperative radiotherapy. The patient declined adjuvant endocrine therapy.

In 1991 and 1994, respectively, the patient had local recurrences in the right breast, found on follow-up mammograms, for which she underwent a lumpectomy followed by simple mastectomy. On both occasions, the patient declined adjuvant endocrine therapy.

In 1998, she presented with stage IV disease consisting of right axillary recurrence with soft tissue skin involvement. She underwent surgical resection, followed by radiotherapy to the axilla and supraclavicular regions consisting of $50 \mathrm{~Gy}$ in 25 fractions. At this point, the patient accepted therapy with tamoxifen and subsequently remained disease free for a period of 7 years.

In 2005, the patient underwent a metastatic workup when she presented with non-specific musculoskeletal pains in her hip and spine. X-rays and MRI of the spine were within normal limits. A positron emission tomography (PET) scan performed at that time did not show fluorodeoxyglucose (FDG) avid lesions, however, multiple minute non-FDG-avid lung nodules were visualised bilaterally. In 2006, repeat MRI of the spine for worsening symptoms revealed multilevel LC picked up at the thoracolumbosacral spine. A dedicated MRI of the brain also demonstrated involvement around the fourth ventricle. The diagnosis was confirmed by cerebrospinal fluid (CSF) cytology where carcinomatous cells displaying the same histopathology as those obtained from the previous axillary recurrence were observed (figures 1 and 2). PET scan also confirmed FDG uptake in the leptomeninges around the spine, without evidence of systemic FDG-avid disease. The patient was started on palliative radiotherapy to the involved areas of her spine, consisting of $30 \mathrm{~Gy}$ in 10 fractions. 


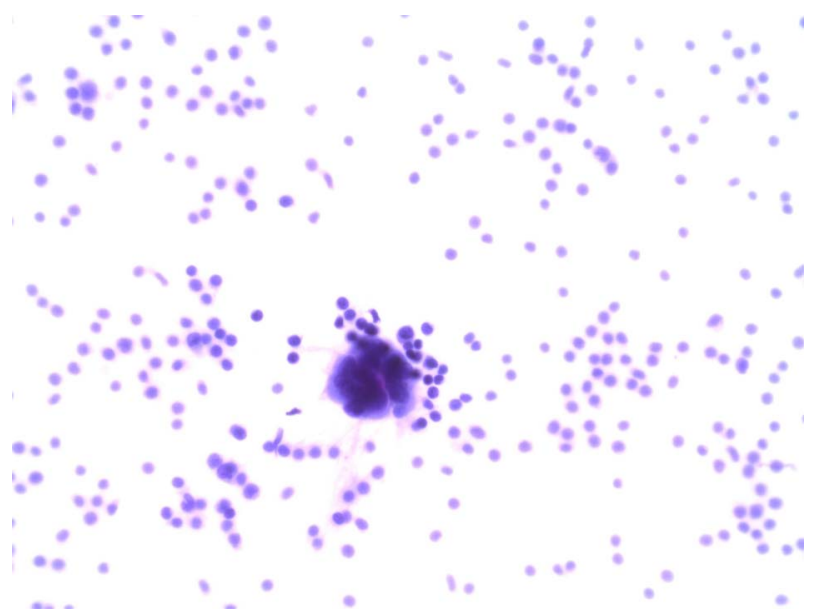

Figure 1 Cerebrospinal fluid cytology: the metastatic adenocarcinoma cells show a high nuclear-to-cytoplasm ratio and pseudogland formation. Background of increased number of lymphocytes (H\&E, $\times 400)$.

The patient subsequently underwent an Ommaya reservoir insertion and was started on weekly ITMx. She had a complete cytological response in her CSF after the first dose of ITMx. Shortly after her fourth dose of ITMx, the patient developed disorientation, confusion, intermittent nausea, vomiting and occasional blurry vision. Brain MRI still demonstrated linear enhancement around the meninges, suggestive of LC. CSF analysis from the Ommaya reservoir did not pick up any malignant cells at that time. The most likely diagnosis then was thought to be methotrexate-induced CNS toxicity, and further treatments were halted. The patient was started shortly after on letrozole. Within 6 months of therapy, the patient had complete resolution of the linear enhancements seen on her previous MRI. For the next 4 years, she did not have any evidence of recurrence on follow-up CNS imaging. Her lung nodules remained stable on PET-CT scans.

In 2010, MRI of the spine performed for low back pain showed a new T10 epidural lesion, for which she received palliative radiation therapy consisting of $20 \mathrm{~Gy}$ in 5 fractions. The patient's letrozole treatment was shortly interrupted for financial reasons, and she was subsequently started on exemestane, which was stopped in mid-July 2011, again for financial reasons. In January 2012, PET-CT scan showed progression of the size of the previously seen lung nodules, now FDG-avid, and newly present 4.5 FDG-avid left adnexal metastatic deposit. The patient

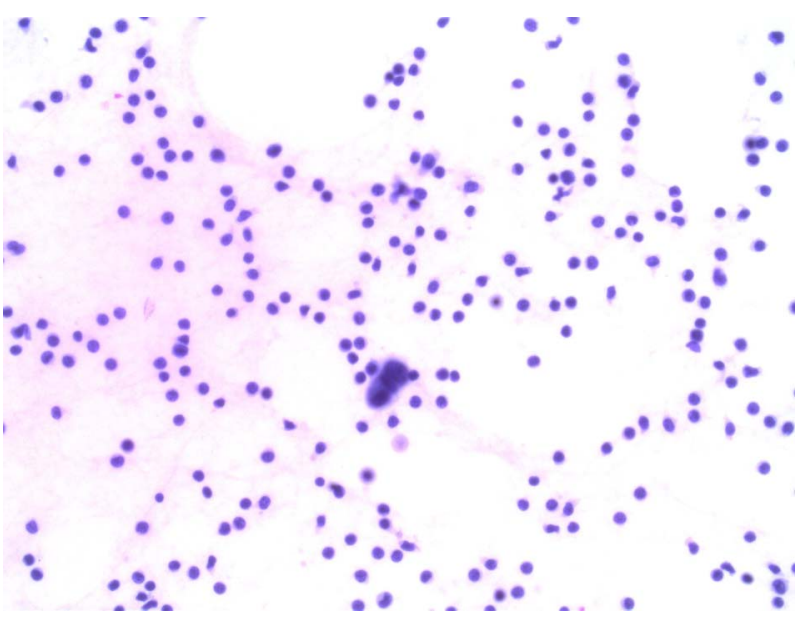

Figure 2 Cerebrospinal fluid cytology: small cluster of three malignant cells, which show increase of cell and nuclear size in rapport with normal lymphocyte cells in background $(H \& E, \times 400)$.

was restarted on exemestane a few months later after resolution of her financial situation. This therapy resulted in stabilisation of her metastatic tumour burden for the next 2 years. In 2014, there was progression of disease with new liver and bone lesions. At that time, the patient was minimally symptomatic with an Eastern Cooperative Oncology Group (ECOG) score of 1 . She was started on megestrol acetate.

The patient remained stable until mid-2015, when she presented with severe abdominal pain and gastrointestinal symptoms, and was found on exploratory laparotomy to have diffuse ischaemic bowel. She passed away shortly thereafter of haemodynamic shock.

\section{DISCUSSION}

LC in the setting of breast cancer carries a poor prognosis and is most often associated with the lobular subtype. Treatment modalities include surgery for obstructive symptoms, radiation therapy, IT therapy and systemic chemotherapy. IT chemotherapy is often limited by distribution and toxicity. The most common toxicities include ventriculitis/arachnoiditis (10-23\% of cases), leucoencephalopathy $(7.5 \%)$ and bacterial meningitis $(3.75 \%)$ associated with the presence of an intraventricular reservoir. ${ }^{3}$

No prospective trials exist examining the role of hormonal therapy alone or with radiation therapy for the treatment of

Table 1 Case reports of antihormonal therapy for breast cancer with leptomeningeal disease

\begin{tabular}{|c|c|c|c|c|c|}
\hline $\begin{array}{l}\text { Age, } \\
\text { years }\end{array}$ & DFI & Prior therapy & Treatment for LC & PFS & Reference \\
\hline 72 & 7 years & FAC $\times 6$, tamoxifen $\times 5$ years, progestin, aminoglutethimide & Formestane & $>2$ years & 5 \\
\hline 66 & 0 & Prednisone & Letrozole & $>1$ year & 6 \\
\hline 32 & 1 year & $\mathrm{CMF}$ & Ovarian ablation/tamoxifen & 10 months & 7 \\
\hline 52 & 9 years & $\mathrm{CMF} \times 6$ & Tamoxifen, local RT & 6 months & 7 \\
\hline 41 & 33 months & CMF×6, tamoxifen, for LC whole brain RT, ITMx, cisplatin-etoposide & Letrozole & 16 months & 8 \\
\hline 34 & 10 years & $\begin{array}{l}\text { CMF, tamoxifen, paclitaxel/trastuzumab docetaxel/epirubicin, ITMx with } \\
\text { etoposide }\end{array}$ & Exemestane & 10 months & 9 \\
\hline 60 & 5 years & $\begin{array}{l}\text { CMF, tamoxifen } \times 5 \text { years } \\
\text { ITMx }\end{array}$ & Letrozole & $>36$ months & 10 \\
\hline 47 & 0 & None & $\begin{array}{l}\text { Tamoxifen/ovarian ablation, letrozole, } \\
\text { everolimus }\end{array}$ & 10 months & 11 \\
\hline
\end{tabular}

CMF, cyclophosphamide, methotrexate, 5-FU; DFI, disease-free interval; FAC, 5FU, adriamycin, cyclophosphamide; ITMx, intrathecal methotrexate; LC, leptomeningeal carcinomatosis; PFS, progression-free survival; RT, radiotherapy. 
oestrogen receptor-positive LC. There is pharmacological evidence that some endocrine therapy agents such as tamoxifen and its metabolites can achieve high concentrations in the CNS, up to 46 -fold higher than serum levels. ${ }^{4}$ Other indirect evidence also suggest that antihormonal agents do actually cross the bloodbrain barrier. Hot flashes, one of the major side effects of such therapies, are thought to be centrally mediated by centres in the hypothalamus and the brain stem. Most hormonal therapies, with the exclusion of fulvestrant, are well known to cause hot flashes and it has been postulated that this effect is achieved by the crossing of the drug or their metabolites past the blood-brain barrier.

Multiple case reports have been published documenting the successful treatment of LC in breast cancer with antihormonal alone. These cases have been summarised in table 1 . All cases had a progression-free survival of at least 6 months, with some well over 24 months, which is longer than the documented average LC from breast cancer. Most of these patients also received prior systemic

\section{Learning points}

- Advances in systemic therapies have resulted in a considerable increase in the number of long-term survivors with advanced breast cancer, resulting in an increased incidence of late-onset central nervous system metastasis.

- Endocrine therapy seems to cross the blood-brain barrier, resulting in a possible sustained response in patients with breast cancer and LC.

- Hormonal therapy for LC in patients with hormone-positive breast cancer is a good treatment option for those with good performance status, minimal hormonal therapy exposure and a low systemic burden of disease not requiring intravenous chemotherapy. chemotherapy for systemic disease control. Our case remains the longest documented progression-free survival of a patient with breast cancer and LC treated with endocrine therapy, and is further evidence that such therapies could be safely used with good efficacy and a safe toxicity profile in a select group of patients.

Competing interests None declared.

Provenance and peer review Not commissioned; externally peer reviewed.

\section{REFERENCES}

1 Mittica G, Senetta R, Richiardi L, et al. Meningeal carcinomatosis underdiagnosis and overestimation: incidence in a large consecutive and unselected population of breast cancer patients. BMC Cancer 2015;15:1021.

2 Le Rhun E, Taillibert S, Zairi F, et al. A retrospective case series of 103 consecutive patients with leptomeningeal metastasis and breast cancer. J Neurooncol 2013;113:83-92.

3 Kwong YL, Yeung DY, Chan JC. Intrathecal chemotherapy for hematologic malignancies: drugs and toxicities. Ann Hematol 2009;88:193-201.

4 Lien EA, Wester $K$, Lønning PE, et al. Distribution of tamoxifen and metabolites into brain tissue and brain metastases in breast cancer patients. $\mathrm{Br} J \mathrm{Cancer}$ 1991;63:641-5

5 Andrés $\mathrm{E}$, Goichot $\mathrm{B}$, Kurtz JE, et al. Carcinomatous meningitis secondary to breast cancer. Spectacular response to hormone therapy. Presse Med 1999;28:75.

6 Staff NP, Bosch EP, Engelstad J, et al. Metastatic lobular breast adenocarcinoma presenting as cauda equina syndrome. J Peripher Nerv Syst 2010;15:75-8.

7 Boogerd W, Dorresteijn LD, van Der Sande JJ, et al. Response of leptomeningeal metastases from breast cancer to hormonal therapy. Neurology 2000;55:117-19.

8 Ozdogan M, Samur M, Bozcuk HS, et al. Durable remission of leptomeningeal metastasis of breast cancer with letrozole: a case report and implications of biomarkers on treatment selection. Jpn J Clin Oncol 2003;33:229-31.

9 Navarro Martín LM, Ocaña Fernández A, Rodríguez Sánchez CA et al. Durable clinical benefit with exemestane in leptomeningeal metastasis of breast cancer. Clin Transl Oncol 2005;7:358-60.

10 Peroukides S, Onyenadum A, Starakis I, et al. Prolonged survival of neoplastic meningitis from breast cancer with letrozole and intrathecal methotrexate: a case report. J Neurooncol 2011;101:509-11.

11 Zoghi B, Elledge R. Endocrine therapy for leptomeningeal metastases from ER-positive breast cancer: case report and a review of the literature. Breast $J$ 2016;22:218-23.

Copyright 2016 BMJ Publishing Group. All rights reserved. For permission to reuse any of this content visit

http://group.bmj.com/group/rights-licensing/permissions.

BMJ Case Report Fellows may re-use this article for personal use and teaching without any further permission.

Become a Fellow of BMJ Case Reports today and you can:

- Submit as many cases as you like

- Enjoy fast sympathetic peer review and rapid publication of accepted articles

- Access all the published articles

- Re-use any of the published material for personal use and teaching without further permission

For information on Institutional Fellowships contact consortiasales@bmjgroup.com

Visit casereports.bmj.com for more articles like this and to become a Fellow 